\title{
At the intersection of materials, engineering, and new business creation
}

\author{
Gregory J. Galvin, Peng Zhou, Timothy J. Davis, \\ Shahyaan Desai, and Shane Collins
}

\begin{abstract}
Engineering, whether in the form of product development or manufacturing processes, often drives the selection or creation of new materials in order to meet performance requirements. Conversely, development of new materials, or new ways to process materials, can lead to new engineering capabilities that, in turn, lead to new products or improved product performance. The interplay between materials and engineering is dynamic, ongoing, and critical to the success of many new products and industries. In this article, we take examples of this interplay from four technology companies in different industries developing widely different materials systems. Each example demonstrates the critical role that materials play in creating new products, new manufacturing methods, and even new design methodologies. Our examples come from polymer microfluidic devices, silicon- and nonsilicon-based microelectromechanical systems, and metals additive manufacturing.
\end{abstract}

\section{Introduction}

Historians, though not having the perspective of materials scientists, have explicitly recognized the importance of materials throughout human history by giving eras names such as the Stone, Bronze, and Iron Ages. ${ }^{1}$ In more recent times, there have been numerous examples of how new materials or ways of manipulating materials have led to advances in engineering that, in turn, created new products and market opportunities. Modern air travel is, in large part, made possible by the capabilities of jet engines, with those capabilities, in turn, largely arising from the alloys used in the turbines that allow for reliable operation at very high temperatures. ${ }^{2}$ Everyday products from optical-fiber-delivered cable television to compact disc players to light-emitting diode light bulbs owe their existence to the invention of the molecular beam epitaxy process that enabled the creation of compound semiconductor materials at the atomic level. ${ }^{3}$ Of great potential, now and in the future, is the field of biomedical engineering, one aspect of which is the use of biomaterials in tissue engineering to replace and repair human organs. ${ }^{4}$

This article examines experiences in four companies in which the authors have been involved, where the intersection of materials and engineering were fundamental to creating the business. Rheonix's microfluidic products are based on the invention of a novel polymer adhesion process. Kionix is able to reliably manufacture high volumes of silicon microelectromechanical inertial sensors because of materials engineering of the surfaces and thin films used in the construction of the products. Mezmeriz solved an inherent tradeoff between speed and displacement of a micro-mirror device by integrating different classes of materials to optimize both performance parameters simultaneously. Incodema3D is exploiting the market opportunity created by additive manufacturing of metals while facing the challenges of materials characterization arising from this new fabrication process.

\section{Microfluidic devices: Materials and processing}

Microfluidic devices have long been considered for economical disease detection and other diagnostic applications. Using small volumes of expensive reagents, leveraging opportunities for low-cost volume manufacturing and enhancing performance through optimum surface-to-volume ratios are some of the many motivations for microfluidic devices.

In the early 2000 s, many microfluidic ${ }^{5}$ devices were fabricated using silicon microfabrication. Although there were market demands for novel features that could be made in silicon, practical applications such as human in vitro diagnostics were hampered by the inability to handle "large" $(1-10 \mathrm{ml})$

Gregory J. Galvin, Rheonix, Inc., USA; ggalvin@rheonix.com

Peng Zhou, Rheonix, Inc., USA; pzhou@rheonix.com

Timothy J. Davis, Kionix, Inc., USA; tdavis@kionix.com

Shahyaan Desai, Mezmeriz, Inc., USA; sdesai@mezmeriz.com

Shane Collins, Incodema3D, LLC, USA; scollins@incodema3d.com

DOl: $10.1557 / \mathrm{mrs} .2015 .232$ 
sample volumes; the difficulty in systems integration; and, most of all, high material and fabrication costs for silicon devices. Rheonix, Inc. was founded to develop low-cost, fully automated molecular diagnostic systems. The company's first challenge was to find an alternative to silicon for its core technology. To address these issues, the Rheonix team looked to polymeric materials. ${ }^{6,7}$

Numerous polymers could potentially be used in this application, and many of them can be joined together by thermal energy or solvents. However, the need to preserve device microstructures and to have a scalable, cost-effective manufacturing process quickly reduced the list of viable combinations of materials and fabrication processes.

\section{Rheonix challenge: A cost-effective, disposable, microfluidic system for molecular diagnostics}

A unique solvent lamination technique ${ }^{8,9}$ developed by Rheonix addresses the microplastic fabrication challenges to meet the biological assay application requirements and the price target for the diagnostic market. The solvent, when applied at room temperature to the plastic substrate, has little effect on the material. When the solvent is sandwiched between two mating surfaces, the surfaces can slide against each other easily to allow feature alignment. A modest increase in pressure and temperature rapidly forms a permanent bond between the two surfaces.

A fundamental challenge in microfluidics is producing enclosed channels at small dimensions. In the lamination technique, grooves machined into one substrate become enclosed channels upon bonding with another planar substrate. For this technique to be viable, it first had to be demonstrated that the process would not compromise the dimensional integrity of the small structures that would ultimately comprise the channels, pumps, and other fluid-handling features. Figure 1 shows test coupons of 1-mm-thick polystyrene substrates with microstructures as small as $2.5 \mu \mathrm{m}$. After $5 \mathrm{~min}$ of exposure to the lamination solvent at room temperature, no structural changes could be observed, as seen in Figure 1b. Other tests demonstrated that by controlling the lamination temperature and pressure, channels of a few micrometers in height could be readily enclosed without structural change.

By applying the solvent lamination process between a thin film of 20-100- $\mu \mathrm{m}$ thickness and a flat polystyrene substrate of 1-mm thickness containing milled microfluidic channels, through-holes for introducing reagents, and structural features for the formation of a functional microfluidic network, a highly sophisticated microfluidic structure can be formed in tens of seconds. In the example shown in Figure 2, the structures formed during solvent lamination comprise fluidic pathways, diaphragm valves, pumps, and a polymerase chain reaction reactor for molecular amplification.

The development of this device illustrates the challenges of materials selection and processing in a highly constrained engineering application. Biochemical compatibilities limit the available choice of materials. Microstructures required for the desired fluid manipulation constrain both materials and fabrication processes. The economics of the market application dictate further limits on the choice of materials and require a scalable, low-cost manufacturing process. Last, the need to integrate the microfluidic device into an overall system in terms of both operation and manufacturing adds yet another set of engineering constraints.

\section{Reliability in high-volume commercial inertial sensors}

Inertial sensors made from microelectromechanical systems (MEMS) have permeated the marketplace during the past decade, growing from a niche market for free fall, or drop, detection to pervasive use in handsets, tablets, and wearable electronic devices. Competition and manufacturing scale have reduced the cost of these devices so much that shipments of inertial MEMS sensors to the consumer and automotive sectors currently exceed one billion units annually, with defect rates approaching those of standard semiconductor integrated circuits.

Accelerometer sensors, typically constructed to measure three axes of linear acceleration, are pervasive in consumer electronics, with adoption rates nearing $100 \%$ in smartphones and tablets. The early performance of accelerometers, however, was impaired by "stiction" both in process (during fabrication) and in use (in the field). Stiction results from attractive forces between surfaces at the microscopic scale that prevent free motion of the mechanical proof mass. It is exacerbated by the smoothness of the surfaces or the charge states present in surface damage layers. ${ }^{10}$ In-process stiction can be circumvented through dry release of parts using reactive-ion etching or vapor-phase etching to avoid liquid surface tension. ${ }^{11}$ In-use stiction, however, remains a critical latent defect and has often been combated with anti-stiction coatings. ${ }^{12}$
Figure 1. Images of test microstructures (2.5-5.0- $\mu \mathrm{m}$ lines and spaces, as indicated) (a) before and (b) after exposure to the lamination solvent. Courtesy of Rheonix, Inc. 


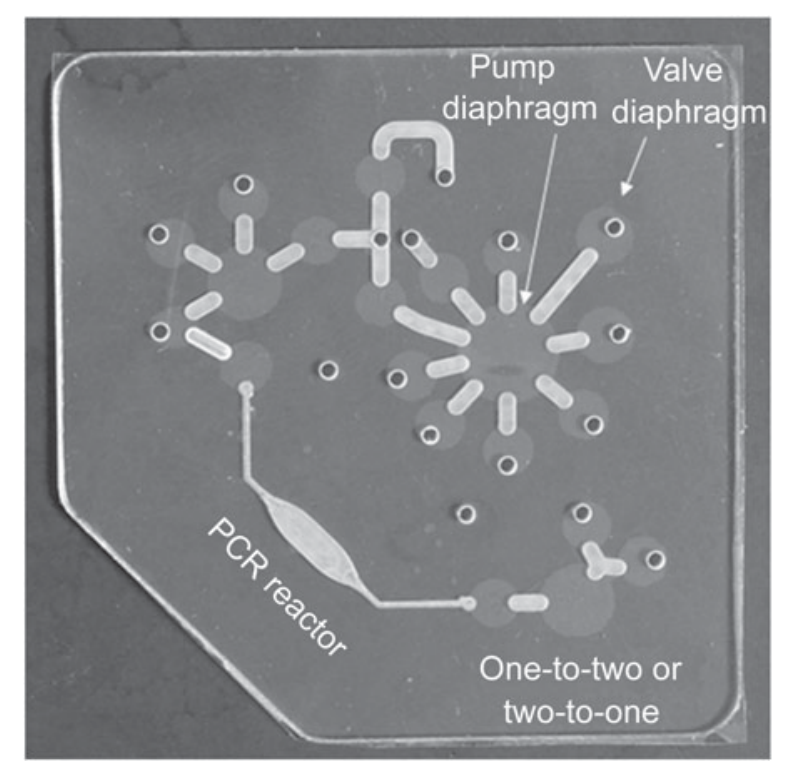

Figure 2. Using the solvent lamination technique, a microfluidic device with valves, pumps, and a polymerase chain reaction (PCR) reactor was fabricated in a single process step. A feature of the technology, shown in the lower right corner, is the ability for bidirectional pumping such that the contents of the one chamber can be pumped into the two chambers at the far right, or the contents of the two chambers at the far right can be pumped into the single chamber between them and the PCR reactor. The total device shown measures about $5 \mathrm{~cm} \times 5 \mathrm{~cm}$. Courtesy of Rheonix, Inc.

The engineering tradeoff between mechanical design and material properties is apparent, as the coating reduces the force that the mechanical design must provide to overcome microstructure adhesion. For a typical low- $g$ accelerometer used in consumer electronics, an anti-stiction coating allows the design to operate with only a few micronewtons of mechanical restoring force.

\section{Kionix challenge: Operation of silicon microelectromechanical inertial sensors}

A practical limitation to anti-stiction coatings, however, is that the applied coating, such as a standard organic monolayer, must not degrade during backend processes such as wafer-level encapsulation. Figure 3 shows the modification of an organic anti-stiction coating, as measured in terms of the contact angle of a water droplet by a goniometer before and after a $420^{\circ} \mathrm{C}$ wafer-bonding thermal cycle. The ability to repel water is a primary criterion for anti-stiction coatings, as increasing levels of water vapor significantly enhance stiction. As shown in the figure, the coating effectiveness clearly degrades as a result of the thermal cycle, so the coating deposition and surface preparation must be engineered to stabilize performance. Neither contact-angle measurements nor more sophisticated analyses of coating material properties such as x-ray photoelectron spectroscopy can perfectly predict anti-stiction performance, however. In practice, an empirical learning cycle among design

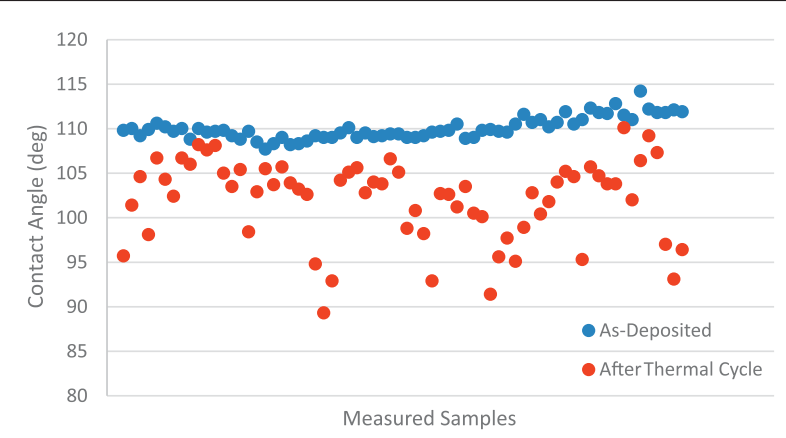

Figure 3. Contact angles of a water droplet on samples of anti-stiction films before and after a wafer-bonding thermal cycle. Courtesy of Kionix, Inc.

variants, film deposition properties, and mechanical testing has been used to optimize device stiction immunity.

Gyroscopes are related to accelerometers in terms of manufacturing processes, but they pose different challenges in materials science. Whereas accelerometers measure linear acceleration, gyroscopes measure rate of rotation about an axis, or angular velocity. They are built using methods similar to those used for accelerometers, but in operation, gyroscopes are excited at their mechanical resonance so that changes in angular velocity result in a Coriolis force that can be detected.

Gyroscopes typically do not have in-use stiction issues because the element mechanical resonance and stiffness are a factor of 10 higher than those of accelerometers. However, because gyro sensors must be driven to oscillation and maintained at amplitude, they are subject to gas damping effects within their microenvironment. Higher vacuum levels increase the gyro quality factor $(Q$, a measurement of the bandwidth of the resonator or, equivalently, the time constant to energize it) and allow the gyro to use less energy to maintain a stable amplitude. The choice of materials comprising the structure is critical for maintaining vacuum levels and ensuring that gas molecules trapped within these materials do not desorb, so that the pressure within the gyroscope cavity is constant and gyro performance is stable.

Gyroscopes can employ a number of different getter materials to capture outgassed substances within their encapsulated environment. ${ }^{13}$ This technique proves highly successful for gettering reactive elements such as nitrogen and oxygen, which can be continuously reabsorbed within the getter, but is ineffective against inert molecules such as argon, which is commonly embedded in sputtered materials and conformally deposited films.

Figure 4 shows outgassing analysis by thermal desorption spectroscopy of a high-density oxide dielectric used in gyro fabrication. At temperatures near $400^{\circ} \mathrm{C}$, many elements start diffusing rapidly from the oxide, including argon, which is used in plasma depositions to improve conformality. Other materials, such as sputtered metals, might outgas argon at a significantly lower temperature. An engineering solution to 


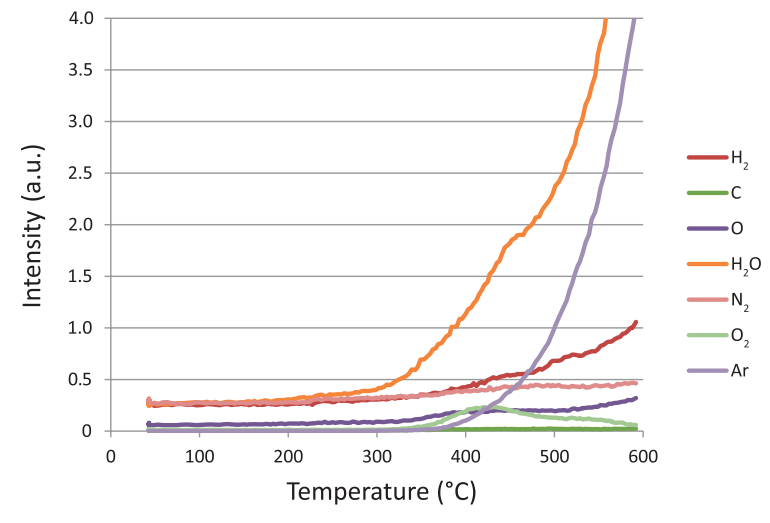

Figure 4. Thermal desorption spectroscopy analysis of gas desorption from a high density oxide deposition film used in gyro fabrication, showing strong desorption around $400^{\circ} \mathrm{C}$ for moisture and a significant increase for argon. Courtesy of Kionix, Inc.

this problem relies on two activities: minimizing the areal coverage of materials that can desorb inert gases and creating barrier layers using highly conformal films deposited by lowpressure chemical vapor deposition or atomic layer deposition. These films effectively act as a gas barrier and trap the inert molecules from diffusing into the gyroscope operating cavity.

In MEMS, no single technique exists for directly measuring either the surface adhesion forces experienced by an accelerometer or the partial pressure in a submillimeter cavity of a gyroscope. By indirectly characterizing the performance of the device and applying the resulting knowledge to the thinfilm properties of the materials in the manufacturing process, the design and fabrication processes are modified to release the product to high-volume manufacturing. For inertial MEMS sensors, this cycle of optimization has contributed to billiondollar industries and supported the revolution in handheld consumer electronics.

\section{Scanning microelectromechanical mirror technologies}

Electrically actuated microelectromechanical mirrors are ideally suited for pico-projector (handheld projector) and threedimensional (3D) scanner applications because the technology lends itself naturally to miniaturization and portable devices. Unlike MEMS mirror arrays, the scanning architecture requires just one or two mirrors to produce a high-resolution display or a $3 \mathrm{D}$ scanner by rapidly scanning a beam in a line along the horizontal direction (fast-scan axis) while slowly scanning the line in the vertical direction. This makes the scanning system inherently more cost-effective than competing panel displays. The simplicity of this design and the potential for low-power actuation mechanisms for the mirrors are also major advantages. However, optimizing performance requires significant engineering tradeoffs in the design and materials selection for mirrors and actuators.
The key parameters of micro-mirrors are the oscillation frequencies and deflection angles along both the fast and slow scan axes (which constrain the scanning speeds and amplitudes) and the mirror size (clear aperture) and its flatness during the scan. Each of these parameters impacts the ultimate resolution and size of the image that the scanning system can project.

The scanning frequency requirement of a micromirror is determined by the number of lines and frame rate desired in the projected image. The nominal scan frequency for video along the slow axis is typically between 30 and $60 \mathrm{~Hz}$. A linear ramp drive is typically desired along this axis so that lines of pixels in each projected frame are scanned sequentially to take advantage of persistence of vision.

The number of lines in the vertical direction multiplied by the frame rate, plus some overhead to allow the mirror to return to its original position and begin the next frame, defines the time available for scanning along the fast (horizontal) axis. For video images of Video Graphics Array resolution (640 $\times$ 480 pixels) and above, this means that a micromirror system must be capable of scanning at tens of kilohertz along the fast or line-scan axis. Physically moving anything, however small, at such frequencies would pose significant challenges in terms of power consumption. However, MEMS mirrors rely on the mechanical gain or $Q$ factor by operating the scanner at or near its mechanical resonance frequency along the fast scan axis.

In addition to high scan speeds, the resolution of images that scanning micromirrors can project depends on their mechanical scan angles along both axes. The number of resolvable pixels in a scanned line improves with larger scan angles, as does the number of resolvable lines in a scanned frame. Large scan angles also result in a wider projection cone, producing images that can be projected within a shorter distance or a larger field of view for a 3D scanner. This is often an overlooked feature; however, it is crucial because it enables interactivity with the projected images and greater detail of the surface to be scanned.

The majority of MEMS scanning micromirrors today rely on silicon as the structural material. Decades of process development from the semiconductor industry have facilitated fabrication and integration of electrical sensing and driving elements directly into silicon micromirrors. Several mirrors can be fabricated on a wafer in a batch process, making manufacturing highly affordable. Silicon's low density and relatively high Young's modulus are important mechanical parameters that can be exploited in the design of MEMS micromirrors. However, silicon's brittle nature and orthotropic mechanical properties, which are often affected by the process conditions, also present significant challenges in the performance of MEMS scanning micromirrors.

A typical ${ }^{14}$ scanning micromirror consists of a circular or rectangular piece of silicon coated with a reflective metal, supported on two opposite sides by collinear silicon beams. It is usually also attached to motion-generating elements that 
use piezoelectricity or electrostatic or electromagnetic forces to twist the silicon beams (called flexures), forcing the circular structure to rotate around the axis created by the beams.

The dimensions of the circular section (which determine the mirror mass and inertia) and the dimensions of the beams (which, along with the Young's modulus of silicon, determine the mirror spring constant) define the resonance frequency of the micromirror. The square of the resonance frequency is proportional to the spring constant and inversely proportional to mass/inertia. For this reason, mirrors are kept as small as possible, typically less than $1.5 \mathrm{~mm}$ in diameter and $0.3 \mathrm{~mm}$ in thickness.

The maximum angle that the mirror can be made to rotate/ scan before it breaks is defined only by the dimensions of the beams. Shorter, thicker beams have higher spring constants but cannot be made to twist very much before they break. Longer, thinner beams can twist through much larger angles but have very low spring constants, preventing the mirrors from scanning at high resonance frequencies. This manifests in micromirror performance as a tradeoff between large scanning angles and faster resonance frequencies, and mechanical excursions exceeding $15^{\circ}$ at frequencies necessary for video projection become virtually impossible. This creates a resolution pseudolimit for pico-projectors and 3D scanning devices using scanning micromirrors.

The challenge lies in extending the limit to which strain energy can be stored in the flexures or beams that cause the mirror to rotate. Ideal mirrors would have beams capable of very large twist angles despite having high spring stiffness, whereas the mirror section itself would have a very low density/ inertia for high scan speeds and be very stiff to maintain flatness during the scan. Virtually no material meets these criteria simultaneously.

\section{Mezmeriz challenge: High scan angle and high scan} speed with a single microelectromechanical mirror A unique method of overcoming this challenge is to combine materials that are suited for different specific functions. Mezmeriz, Inc. has developed such a MEMS technology whereby traditional silicon MEMS technology is married to fibrous materials to form a composite MEMS micromirror, as shown in Figure 5. Silicon, with its near-ideal stiffnessto-weight ratio, forms the mechanical structure for the mirror. The ease with which mirror position-sensing elements, motion-generating structures, and electrical circuitry can be incorporated within silicon also makes silicon well-suited to form other nonmechanical elements of the mirror. The fibrous materials, with their ability to store vastly more strain energy than silicon, are used to form the flexures that allow the mirrors to rotate.

Chemical and physical surface treatments are performed on both the fibrous materials and the silicon to enhance the bonding properties of each surface. A simple joining process using adhesives tailored to bond the fibrous materials to the silicon is used to create the composite MEMS devices, ensuring that

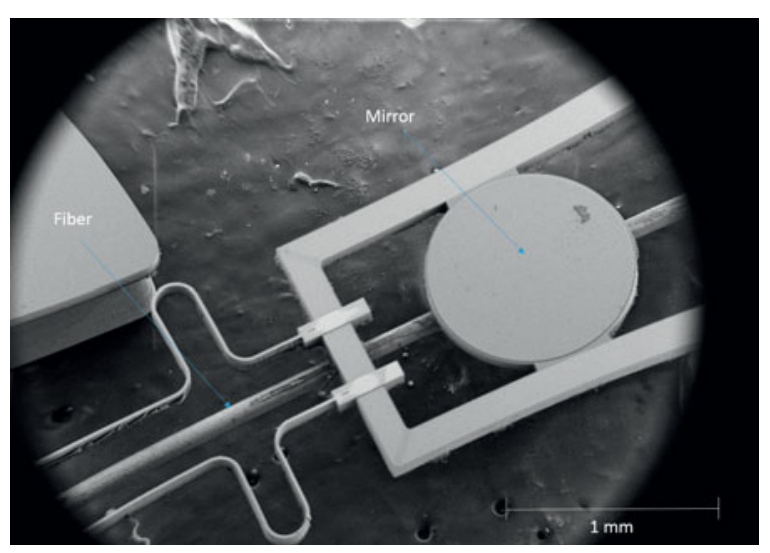

Figure 5. Micromirror assembly with silicon mirror and fiber flexures. Courtesy of Mezmeriz, Inc.

the fibrous materials are securely anchored to the silicon and can withstand extended operation without fatigue.

Such composite MEMS micromirrors retain the low mass and high stiffness of silicon, enabling mirrors that can resonate at high frequencies and maintain flatness during oscillations as well. The ability of the fibrous flexures to undergo high strain despite having high spring stiffness results in mirrors that are capable of large angular deflections at high frequencies.

The development of scanning mirrors that combine different materials systems permits novel near-field projection and hand-held high-resolution 3D scanner configurations without the need for expensive and bulky optics, enabling unique visual interfaces that are more user-friendly and interactive.

\section{Interaction of materials and engineering in metal powder bed fusion AM}

Additive manufacturing (AM), in which parts are "grown" in a layer-by-layer deposition process, has been under investigation and in limited commercial use for several decades. However, recent advances in technologies for AM, particularly for metals, and the availability of new materials have spurred a dramatic increase in the commercial use of AM and moved it much closer to mainstream manufacturing.

According to the Wohlers Report, an annual update on the state of the AM industry, ${ }^{15} 200$ metal AM machines were sold in 2012 worldwide, indicating double-digit growth since 2010. At the 2014 Metal Powder Industries Federation (MPIF) World Congress on Powder Metallurgy and Particulate Materials, Richard Pfingstler, President of MPIF, said that, not counting titanium alloys, metal powders used in additive manufacturing reached $100,000 \mathrm{lbs}(45,000 \mathrm{~kg})$ per year in North America. In July 2014, GE Aviation announced a new $300,000-\mathrm{ft}^{2}\left(28,000-\mathrm{m}^{2}\right)$ plant in Auburn, Ala. that will manufacture fuel nozzles for its next-generation LEAP and GE9X commercial airplane engines using metal powder bed fusion (mPBF), in which a scanned ytterbium fiber laser locally sinters a metal powder. ${ }^{16}$ 
Incodema3D challenge: Characterize and qualify materials

Additive manufacturing is beginning to demonstrate many of the advantages it brings to manufacturing complex geometries, and as a result of diligent research activities, a wide variety of alloys can now be used. Incodema3D, a contract $\mathrm{mPBF}$ manufacturer, offers 11 standard alloys for use in AM, from titanium to high-nickel superalloys to martensitic stainless steels and aluminum. ${ }^{17}$ The ability to 3D-print parts with interesting alloys represents substantial forward progress, but unless the component design is considered, the full benefit of mPBF cannot be achieved.

Consider the following example: A designer creates a simple bracket to allow two surfaces to be supported using conventional design methods as taught in school and reinforced in a commercial setting. The resulting bracket, designed to accommodate the expected loading conditions according to finite-element analysis and data from a material properties database, is shown in Figure 6a. In contrast, Figure 6b shows a topology-optimized bracket whose far more complex shape requires $40 \%$ less mass of the same material to bear the same load as the conventional bracket. This is an example where the $\mathrm{mPBF}$ process and design merge to produce higher-performance components, whereas with conventional manufacturing, a higher-performance component would typically require a new material. mPBF makes it possible to engineer metal foams and lightweight structures analogous to those that have evolved in nature, such as trabecular (spongy) bone for applications that have historically used I-beams and columns. This represents a paradigm change in both materials engineering and design, but there are challenges.

Welding engineers have known for many decades that weld joints pose particular problems because of the inability to subject them to nondestructive testing (NDT) and to estimate their mechanical and stress states. For these reasons, many
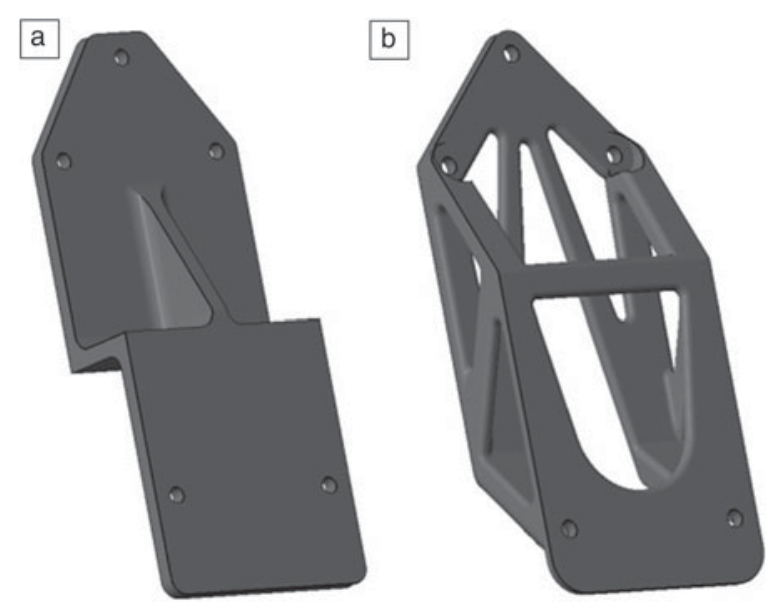

Figure 6. (a) Bracket designed for conventional subtractive machining or casting. (b) Topology-optimized bracket to withstand the same loading conditions with less material. Courtesy of Incodema3D, LLC. safety-critical components are not allowed to be welded for repair or assembly. $\mathrm{mPBF}$ is a continuous welding process wherein a $10-\mathrm{mm}$ cube can contain more than $250 \mathrm{~m}$ of weld tracks, meaning that the cube itself, the cube microstructure, and the cube mechanical properties are all created in a single process. Not only is NDT of such a structure a challenge, but conventional defect characterization does not contain terminology that describes mPBF microstructures. Further, it is possible to alter the microstructure by changing the in-process parameters to achieve, for example, a graded modulus across a component's length. The ASTM E07 Committee on Nondestructive Testing, and specifically subcommittee E07.10, recently started drafting a standard guide on $\mathrm{NDT}^{18}$ for $\mathrm{mPBF}$ materials and will begin to provide guidance in this area in the coming months.

The Incodema3D mPBF materials chart in Reference 17 shows that the chemical compositions of these materials can consist of standard wrought, cast, and forged alloys. These alloys have not been optimized for the mPBF process to reduce residual stress or take advantage of fine microstructure. Rather, these alloys are used because designers are familiar with them and are reluctant to try new materials. In addition, there are no public databases of mechanical property information from which engineers can derive design-allowable curves for mPBF materials. Developing these databases for all possible alloy combinations would take decades and hundreds of millions of dollars. For this reason, mPBF material and process modeling has been made a US national priority, and on that front, progress is being made. King et al. ${ }^{19}$ at Lawrence Livermore National Laboratory reported initial findings about defects related to the transition from "conduction" mode to "keyhole" mode, indicating that weld depth penetration is much deeper than laser energy density would otherwise dictate. Keyhole mode was shown to produce porosity voids in the wake of a laser beam as it passes over the metal powder at velocities exceeding $1000 \mathrm{~mm} / \mathrm{s}$.

$\mathrm{mPBF}$ has been in development for over a decade, but only in the past few years has it been able to process interesting alloys for high-end applications in the aerospace, oil and gas, and medical implant sectors. As an industry, the mPBF community has only begun to investigate the NDT and microstructure and defect detection required for long-term acceptance. Process modeling is emerging and will be necessary to screen candidate alloys specific for $\mathrm{mPBF}$, and future machine designs with, for example, high-temperature chamber capability will produce new materials such as intermetallics and ceramics. With $\mathrm{mPBF}$, the component and system design must be considered holistically for an optimized design.

\section{Summary}

Tradeoffs in materials selection, manufacturing processes, engineering design, and product performance are fundamental in the development of new products and the creation of new markets. In each of the four examples presented in this article, materials engineering was fundamental to the company attaining 
its desired product. Cost considerations drove Rheonix to select polymer materials that, in turn, required the invention of a new adhesion process to attain the desired microfluidic structures. Kionix product reliability requirements dictated the selection of materials and the manner in which they could be processed in high-volume manufacturing. To solve seemingly contradictory product performance requirements, Mezmeriz chose to incorporate both silicon and nonsilicon materials into its micromirror system. Improvements in the types of materials that can be utilized in additive manufacturing have created large new market opportunities for Incodema3D, but significant materials characterization efforts are still required before customers will embrace this new technology. These companies, three still in their formative stages and one (Kionix) that has matured into an industry leader, are all predicated on solving basic materials engineering issues.

\section{References}

1. S.L. Sass, The Substance of Civilization: Materials and Human History from the Stone Age to the Age of Silicon (Arcade Publishing, New York, 2011).

2. J.H. Perepezko, Science 326, 1068 (2009).

3. M.B. Panish, Science 208, 916 (1980).
4. R. Langer, Science 260, 920 (1993).

5. T. Vilkner, D. Janasek, A. Manz, Anal. Chem. 76, 3373 (2004).

6. Z. Chen, G.M. Mauk, J. Wang, W.R. Abrams, P. Corstjens, R.S. Niedbala, D. Malamud, H.H. Bau, Ann. N.Y. Acad. Sci. 1098, 429 (2007).

7. R.H. Liu, J. Yang, R. Lenigk, J. Bonanno, P. Grodzinski, Anal. Chem. 76, 1824 (2004).

8. P. Zhou, L.C. Young, "Laminated Microfluidic Structures and Method for Making," US Patent 7,608,160 (October 27, 2009).

9. L.C. Young, P. Zhou, "Microfluidic Pump and Valve Structures and Fabrication Methods," US Patent 7,832,429 (November 16, 2010).

10. R. Maboudian, R.T. Howe, J. Vac. Sci. Technol. B 15 (1), 1 (1997)

11. Y.-I. Lee, K.-H. Park, J. Lee, C.-S. Lee, H.J. Yoo, J.Y. Kim, Y.-S. Yoon, J. Microelectromech. Syst. 6, 226 (1997).

12. W.R. Ashurst, C. Carraro, R. Maboudian, IEEE Trans. Device Mater. Reliab. 3, 173 (2003).

13. R. Ramesham, R. Kullberg, J. Micro Nanolithogr. MEMS MOEMS 8, 031307 (2009).

14. S.T.S. Holmstrom, U. Baran, H. Urey, J. Micro electromech. Syst. 23 (2), 259 (2014)

15. T.T. Wohlers, "Wohlers Report 2013: Additive Manufacturing and 3D Printing State of the Industry Annual Worldwide Progress Report" (Wohlers Associates, Inc., Fort Collins, CO, 2013)

16. M. LaMonica, MIT Technology Review 116, 58 (2013).

17. "IncodALLOY'M I3D8.1 Materials Chart" (Incodema3D LLC, Freeville, NY, 2015) 18. "New Guide for Nondestructive Testing of Additive Manufactured Metals Parts Used in Aerospace Applications" (Work Item ASTM WK47031, ASTM International, West Conshohocken, PA, 2014)

19. W.E. King, H.D. Barth, V.M. Castillo, G.F. Gallegos, J.W. Gibbs, D.E. Hahn, C. Kamath, A.M. Rubenchik, J. Mater. Process. Technol. 214, 2915 (2014).

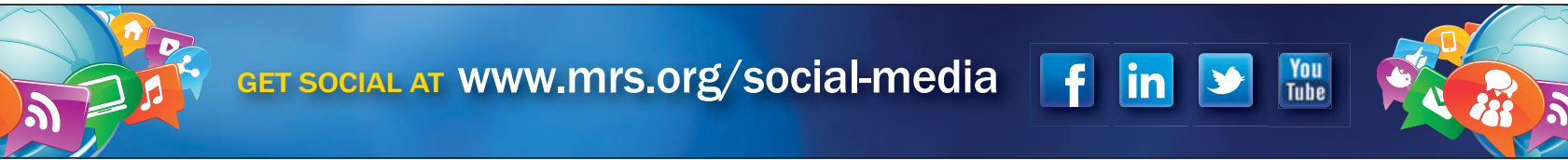

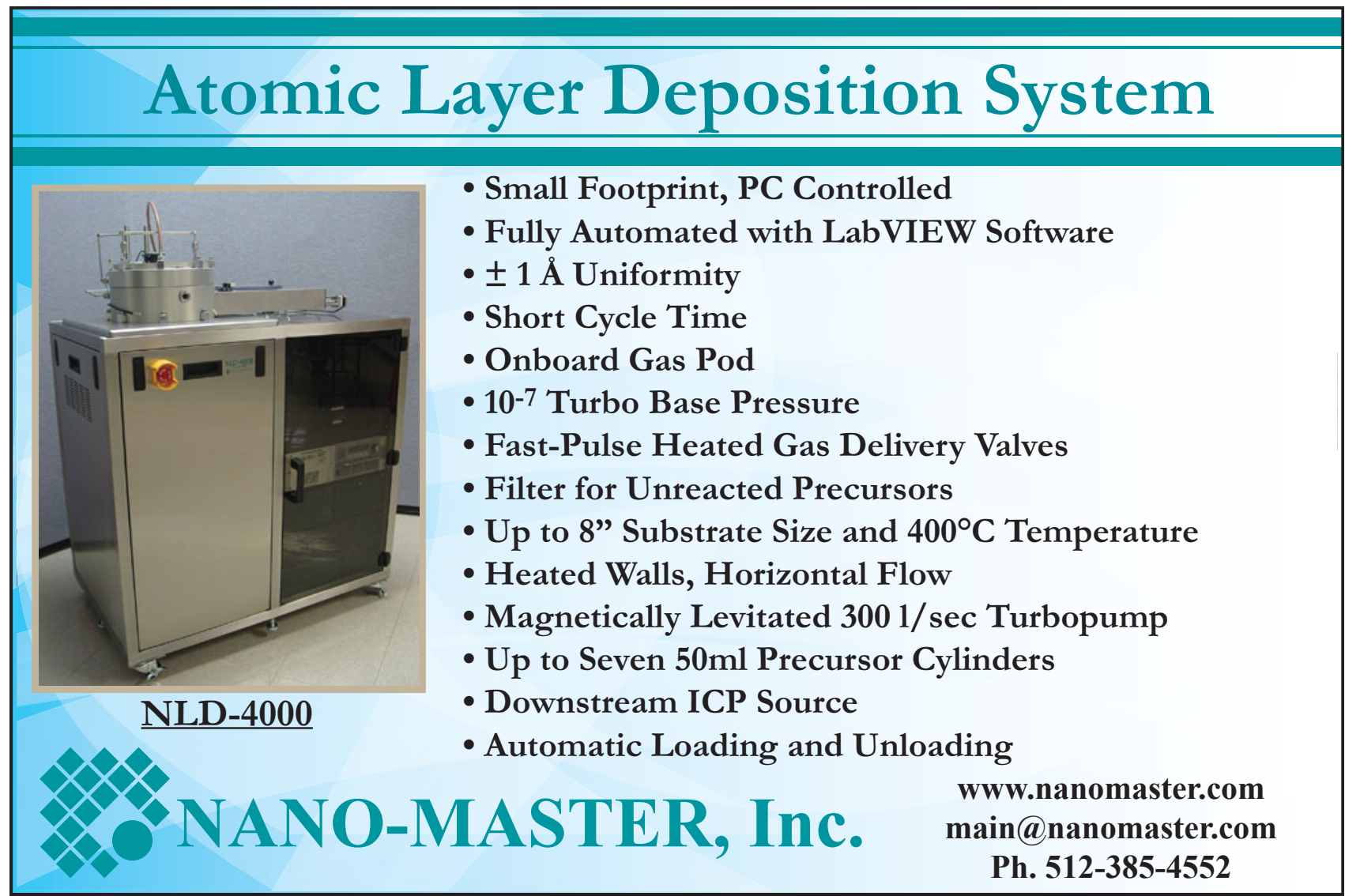

